\title{
Optimization of SU's revenue based on price compensation for collision
}

\author{
Huang Huan, Li Xuxi, Zhang Xiuxiu, Luo Jing, Zhang Fengyang, Xie Gang \\ School of Information and Communication Engineering, Beijing University of Posts and Telecommunications \\ BUPT \\ Beijing, China \\ huanghuan@bupt.edu.cn
}

\begin{abstract}
In the cognitive radio network, when the primary user doesn't use its licensed spectrum, the secondary user can access the spectrum hole opportunistically to share the spectrum. However, if the secondary user's behaviors cause collisions with the primary user in spectrum band, which may lead to a decline of primary user's QoS, the initiative of the primary user to participate in spectrum sharing will be seriously affected. The development and promotion of cognitive radio technology will be hindered once lack the primary user's support. In our scene, in order to reduce the impact of collisions with the primary user, before the secondary user access the spectrum, spectrum sensing must be used to make sure the primary user is not using it. However, most studies about the pricing framework are based on perfect sensing. But in practical network, the result of the spectrum sensing is likely to be wrong. And the probability of sensing errors is affected by the sensing time. In our algorithm, it is taken into account that once sensing errors happen and result in collisions, the secondary user should compensate for the primary user's losses. Therefore, in this paper, we discuss the selection of the sensing time in a cognitive cycle, aiming at optimizing the secondary user's revenue based on price compensation for collision. The signal is transmitted by a frame and a frame. So we divide the spectrum usage time into cognitive cycles, which greatly reduces the complexity of the algorithm. Eventually we calculate the optimal sensing time to make the secondary user's revenue maximized.
\end{abstract}

Keywords-cognitive radio; spectrum sharing; pricing framework; sensing time.

\section{INTRODUCTION}

In cognitive radio networks, a primary user (PU) can transfer data in its licensed spectrum band. It is legal for it to using spectrum without affected by any other unlicensed user. However, the secondary user (SU) has no spectrum license. Therefore, if a secondary user (SU) wants to transfer data, it must share the spectrum band with the licensed PU without interfering with the PU.

In cognitive radio networks, a variety of dynamic spectrum access ways are probably divided into three categories models: dynamic exclusive use model, open sharing model, and hierarchical access model. In hierarchical access model, two approaches to spectrum sharing between primary and secondary users have been considered: spectrum Underlay model and spectrum Overlay model. [1]
When the system is operating in the spectrum Underlay mode, it allows the PU and the SU to transmit data simultaneously in the same band. But the interference between the PU and the SU must be below a certain threshold, the threshold is called interference temperature [2].

In the Overlay model, the PU and the SU are not allowed to transmit data simultaneously in the same band. When the PU doesn't operate in licensed spectrum band, the SU is allowed to operate in the unlicensed spectrum. Therefore, the SU must sense the radio spectrum environment on a regular basis. When the spectrum isn't being used, it can opportunistically transfer data over the spectrum holes left available by the PU, and avoiding collision with active PU.[3]

In this paper, we discuss the issue in the Overlay mode. In our scenario, the SU opportunistically access PUs' spectrum only when the PU is not using the spectrum.

The development of cognitive radio technology needs the support of the PU. There is a useful tool to solve this problem, named spectrum trading. In spectrum trading process, pricing framework is a key issue of interests to both primary and secondary users. [3]Therefore, spectrum pricing framework has become a hot issue.

In [4] and [5], the authors establish a spectrum trading market, PUs compete with each other and adjust prices according to supply and demand. In [6], in order to maximize spectral efficiency, the authors increase the above-mentioned spectrum market and develop a new utility function.

However, most of the spectrum pricing frameworks assume that the spectrum sensing is perfect, the SU only needs to pay the cost of leasing spectrum to the PU. And it ignores the impact on PUs' revenue caused by the error in sensing and the collision between the PU and the SU. In the actual scenario, errors may occur in spectrum sensing. The error can result in a collision between the PU and the SU, which will cause a loss of interests of the PU. So we consider improving the pricing framework to compensate the PU for its loss.

Therefore, in this paper, it is considered that the collisions due to the error in sensing have an influence on the pricing framework. And it makes the scene more reasonable.

Scholars have also carried out extensive research about the spectrum sensing. In [7], the authors discuss the calculation 
method of error probability in the energy detection process in detail. In [8] and [9], the authors consider the SU is allowed to use the spectrum band only when it is not used by the PU. So periodically spectrum sensing is needed. It focuses on the relationship between the detection time and channel efficiency and the throughput of the secondary network. In our model, we study the sensing time's impact on SU's final revenue, considering the length of the spectrum sensing can affect the probability of error. And the pricing framework is based on the compensation to the collision.

Under the established pricing framework, the SU's behavior will affect its own revenue. In [10], the authors provide a self-enforcing spectrum sharing game. And SUs improve their long-term profits by creating cooperation actively.

In [11], the ON / OFF state of the PU is a random process and appears alternately. The duration of occupying channel and exiting channel obey index distribution. The author studies optimal time of a cognitive cycle which makes SU's revenue maximized. In this paper, the channel utilization time is divided into cognitive cycles. And finally SU's revenue is due to the probability of sensing error, not to the influence of the duration of occupying channel and exiting channel of the PU. Thus, the model has been greatly simplified and the complexity of the algorithm is greatly reduced. Besides, the algorithm also makes the scene more realistic by defining the objective function is the allocation of sensing time and transmission time within a determined period.

We consider the optimization of SU's revenue based on price compensation for collision. In practice, the signal is transmitted by a frame and a frame. The channel utilization time is divided into cognitive cycles. In each cognitive cycle, spectrum sensing is conducted in the front part of the time and after that the SU can transfer data. Once the sensing error and spectrum collision happens, the SU should compensate the PU. The greater the probability of sensing errors, the more amount of compensation should be paid. The time of spectrum sensing will affect the probability of error and affect the amount of compensation for the PU, finally affect the SU's revenue. Thus, in a cognitive cycle, the allocation of sensing time and transmission time will affect the SU's final revenue. This paper based on price compensation for collision, discusses the optimal sensing time to maximize SU's total revenue. The channel utilization time is divided into cognitive cycles, which makes the complexity of the algorithm greatly reduced. Besides, the algorithm also makes the scene more realistic by defining the objective function is the allocation of sensing time and transmission time within a determined period.

Our major contributions are as follows:

1. Take the compensation for PU into consideration when the spectrum collision happens. Introduce the scenario of wrong sensing result into pricing framework, making the pricing framework more reasonable and practical.

2. Take the real condition into consideration in which signal is transmitted on a frame-by-frame basis and simplify the model to a time allocating problem of the sensing time and transmission time in a cognitive cycle. Eventually, the SU's revenue is depended on the probability of sensing error caused by different sensing time. And it has nothing to do with the length of time whether PU is occupying the channel or is not in the channel. As a result, the model is significantly simplified.

3. Starting from SU's point of view, we study the allocation of spectrum sensing time for SU to reach its maximum revenue based on price compensation for collision

The rest of the paper is arranged as follows:

The second part is an introduction of the system model and hypothesis. The specific logic and formulas are presented in the third part. The fourth part presents the simulation result and the effect of some parameters on the objective function. In the last part, we draw the final conclusion.

\section{SySTEM MODEL}

We now detail the system model. In a cognitive radio spectrum sharing network, the PU leases its spectrum to the SU. We assume that there exists a relationship of leasing spectrum only between a single PU and a single SU. Our scenario model is the Overlay model, in which the SU periodically senses spectrum and detects the PU's spectrum occupancy. If the SU finds a spectrum hole, the SU will access it. The SU avoids collisions with the PU who is in the process of using the spectrum through sensing-transmission strategy. In other words, in our scene only when the SU senses the PU not to use its spectrum, can the SU has a chance to access it.

In the communication system, the signal is transmitted by a frame and a frame. Therefore, spectrum sensing of the SU can be conducted on the cognitive cycle for the unit. Therefore, we assume that the time of a cognitive cycle is $T$, which the first time $t$ for spectrum sensing, the rest of the T-t time for data transmission (Fig.1).
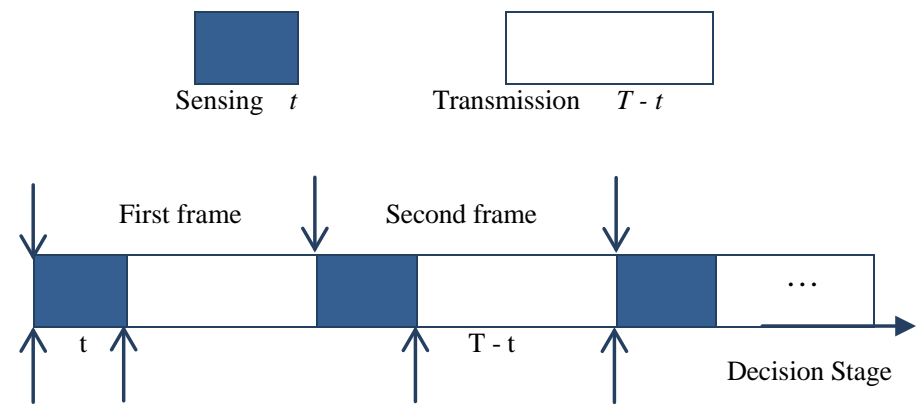

Fig. 1 The Scene of Spectrum Sensing and Sharing

In the actual process, if the sensing time $t$ is too short, the probability of spectrum sensing error will be greater and the possibility of the collision between the PU and the SU will also be greater, thus, the compensation for collisions costs will be greater. On the other hand, if $t$ is too long, although the probability of sensing errors will drop, the SU's throughput is reduced. Thus, the proportion of sensing time needs to be determined according to the revenue.

We assume that the signal $H_{0}$ indicates the PU doesn't send a signal which means the spectrum is not being used. Meanwhile, the signal $H_{1}$ represents that the PU is using the 
spectrum. Only when the SU's sensing results is $H_{0}$, can it have access to the spectrum. If there are some errors with the spectrum sensing, a collision will occur when the SU access the spectrum. Therefore, in this algorithm, the SU not only has to pay the cost of leasing the spectrum, but also needs to pay compensation for the cost of causing collision with the PU. If the SU causes collision with the PU, it needs to compensate for the additional costs $C_{C C}$. Thus, the cost $C$ of the SU to lease the PU's spectrum is divided into two parts: the cost $C_{A}$ to access the spectrum, which is a fixed value; the additional cost $C_{C C}$ to compensate the loss of the PU for the collision caused by the SU, which is related to the accuracy and the time of spectrum sensing. Consequently, the total cost of the SU to lease the PU's spectrum is

$$
C=C_{A}+C_{C C}
$$

\section{ALGORITHM}

\section{A. Collision compensation}

The SU accesses channel when the sensing result is that the PU is not use the spectrum. Unfortunately, the actual spectrum sensing is not fully ideal. For the case when the PU is using its licensed spectrum while the error happens in the SU's spectrum sensing. As a result, the access of SU will cause collision with the PU. The collision will make the PU suffer loss and the loss will result in the SU's total revenue reduced as a punishment. The loss caused by collision is[11]:

$$
(T-t)\left(R_{P}-R S P\right) \alpha^{(T-t)}
$$

Therefore, we define the compensation cost is:

$$
C_{C C}=\theta_{C C} \cdot \Phi_{1}(t)(T-t)\left(R_{P}-R_{S P}\right) \alpha^{(T-t)}
$$

Where, $R_{P}$ is the transmission rate of the PU when it occupies spectrum alone, $R_{S P}$ is the transmission rate of the PU when it suffers from SU's collision, $\theta_{C C}$ is a "price-loss" conversion ratio associated with the utilization of the current spectrum, $\Phi_{1}(t)$ is the probability of collision and $\alpha$ is the compensation factor which is set referring to the impact caused by interference. When $\alpha$ is bigger, it denotes the cost in collision greater and the SU has to spend longer sensing time $t$ to improve accuracy.

In the paper[12],the author studies optimal time of a cognitive cycle which makes SU's revenue maximized based on price compensation for collision. And the ON / OFF state of the PU is a random process and appears alternately because the channel utilization time is not divided into cognitive cycles. And we simplify the scene and set the independent variable more reasonable.

In the process of spectrum sensing, the energy of sample signal detected by the SU is

$$
P=|h|^{2} \sum_{i=1}^{N}\left|X_{i}\right|^{2}
$$

in which $x_{i}$ is transmission signal and $h$ is fixed channel gain. There we set a threshold $\eta$, if $P \geq \eta$, it means the PU is using the spectrum. And this situation is represented by $H_{1}$. On the other hand, if $P<\eta$, it means the spectrum is not used. And this situation is represented by $H_{0}$. To simplify the algorithm we assume the time of a cognitive cycle $T$ submitting to Gaussian distribution. The signal $P d$ represents the probability of the SU sensing the PU is using the spectrum and actually it is correct. And the false-alarm probability $P_{f}$ represents the probability of SU sensing the PU is using the spectrum while actually it is false. Thus they are given by:

$$
\begin{gathered}
P_{d}(t)=P\left(P \geq \eta / H_{1}\right)=Q\left(\frac{\eta-E\left(P / H_{1}\right)}{\sqrt{\operatorname{Var}\left(P / H_{1}\right)}}\right) \\
P_{f}(t)=P\left(P \geq \eta / H_{0}\right)=Q\left(\frac{\eta-E\left(P / H_{0}\right)}{\sqrt{\operatorname{Var}\left(P / H_{0}\right)}}\right) \\
Q(\eta)=\frac{1}{\sqrt{2 \pi}} \int_{\eta}^{\infty} e^{-x^{2}} d x \\
\Phi_{1}(t)=1-P_{d}
\end{gathered}
$$

\section{B. Utility function of $S U$}

The revenue SU gets from leasing spectrum is the data transmission revenue minus the costs which including fixes access costs $C_{A}$ and collision compensation costs $C_{C C}$. The general revenue is given by:

$$
\left.\theta_{G I} \cdot\left[\left(1-P_{f}(t)\right) \cdot R s+\left(1-P_{d}(t)\right) \cdot R P S\right)\right] \alpha^{(T-t)}
$$

Where, $\theta_{G I}$ is a "price-transfer volume" conversion ratio, $R s$ is the transmission rate of the $\mathrm{SU}$ when it leases and occupies spectrum alone and RPS is the transmission rate of the SU when it have collision with the PU. The signal 1- $P_{f}(t)$ represents the probability of the SU sensing the PU not using spectrum and actually correct. And in this situation, the SU can occupy spectrum alone for data transmission without collision with the PU. The signal $1-P_{d}(t)$ represents the probability of the SU sensing the PU not using spectrum but actually false. And in this situation, the SU sharing the spectrum with the PU and transmitting data at a relatively low rate.

Thus, the total revenue of the SU is:

$$
\begin{aligned}
& \left.g(t)=\theta_{G I} \cdot\left[\left(1-P_{f}(t)\right) \cdot R_{S}+\left(1-P_{d}(t)\right) \cdot R_{P S}\right)\right] \alpha^{(T-t)}-C \\
& \left.=\theta_{G I} \cdot\left[\left(1-P_{f}(t)\right) \cdot R_{S}+\left(1-P_{d}(t)\right) \cdot R_{P S}\right)\right] \alpha^{(T-t)} \\
& -\left[C_{A}+\theta_{C C} \cdot\left(1-P_{d}(t)\right)(T-t)\left(R_{P}-R_{S P}\right) \alpha^{(T-t)}\right]
\end{aligned}
$$

It can figure out the optimization sensing time $t$ for maximizing the total revenue of the SU through calculating:

$$
\partial g(t) / \partial t=0
$$

However, it is a transcendental equation and can't be solved directly. So we obtain the numerical solution of the transcendental equation.

As the picture Fig.2 shows, general transmission power of a SU or a PU is under $36 \mathrm{dBm}$. In the case of $\mathrm{T}$ is $100 \mathrm{~ms}$ we 
make the simulation of SU's revenue changing with sensing time . We can acquire the optimal at $32 \mathrm{~ms}$.

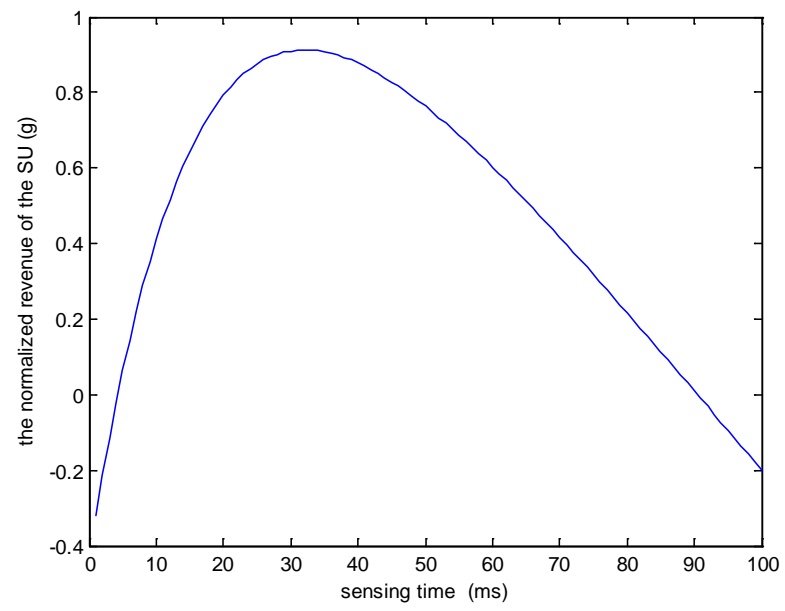

Fig.2 The Normalized Revenue of The SU

\section{Algorithm analysis}

In the algorithm, the final revenue of the $\mathrm{SU}$ is merely relevant to the false probability of sensing and not caring about how long the PU access or release the channel, so we simplify the model greatly. Apparently, the time of sensing makes a difference to system average throughput directly. The SU will not transmit data in sensing time. And if the sensing time is too long, it would reduce the utilization of the SU while too short it would increase the probability of sensing error and cause collisions between SU and PU. Finally, through the game we get optimal sensing time to maximize the revenue of the SU based on price compensation for collision.

\section{SimUlation RESULTS}

In the simulation, we specify a certain channel in a cognitive network model and analysis the effect of the strategy. It's assumed that the PU's and SU's common transmission power are both limited to $36 \mathrm{dBm}$ and the time of a cognitive cycle is $T=100 \mathrm{~ms}$. And the channel noise is white Gaussian noise.

The curve in Fig. 2 shows SU's effectiveness with the perception time. The compensation factor $\alpha$ is set to 1.05 . In the simulation, we have taken 100 sampling points in the unit length of sensing and calculated the average utility of the 100 samples. It can be seen from the statistical results as is shown in Fig. 2, the SU's revenue change with the sensing time is a unimodal distribution, and the optimal solution is reached at $t$ $=32 \mathrm{~ms}$.

It can be seen from the figure that when the spectrum sensing time is very short, the probability of sensing errors is high, thus the compensation for the collision with the PU is great, which makes the total revenue negative. When the spectrum sensing time is very long and the SU's transmission time is short, consequently less profit of transmission will be obtained, therefore the total revenue will also be negative. In the process of increasing the sensing time, the SU's revenue from data transferring gradually reduces, because the compensation costs due to collisions gradually reduced. The total revenue first increased then declined, eventually reached the optimum at $t=32 \mathrm{~ms}$.

Afterwards, we analyzed the influence of several parameters in the formulas on the objective function.

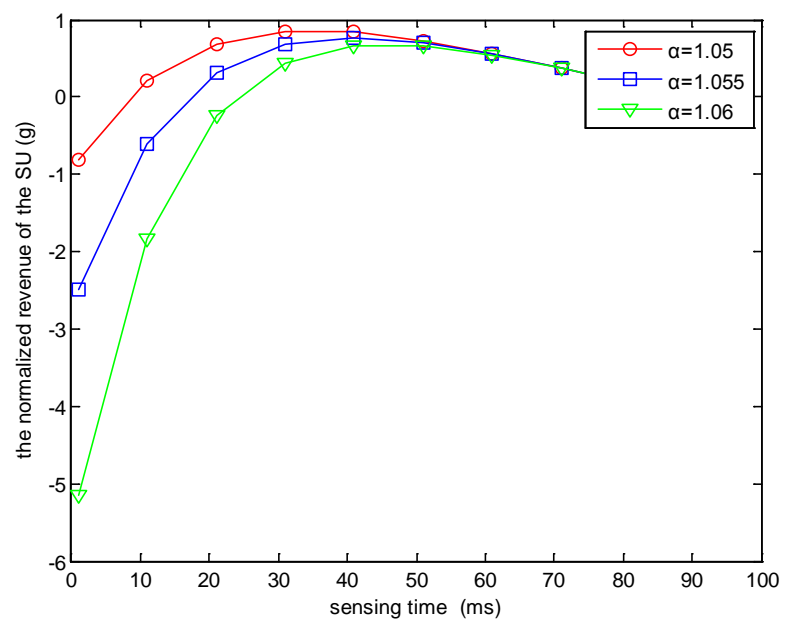

Fig.3.Different $\alpha$ 's Influence on the SU's Revenue

In Fig. 3, the three curves show the SU's revenue when the compensation factor is set to different values. Compensation factor represents the PU's endurance with the length of collision time (BER, packet loss rate and retransmit rate, etc.). In the situation of $\alpha=1.05$, the optimal sensing time is $t 1=32 \mathrm{~ms}$. In the situation of $\alpha=1.055$, the optimal sensing time is $t 2=38 \mathrm{~ms}$. In the situation of $\alpha=1.06$, the optimal sensing time is $t 3=43 \mathrm{~ms}$. As the increase of compensation factor, longer sensing time will result in the smaller compensation cost, optimal sensing time increases as compensation factor increases.

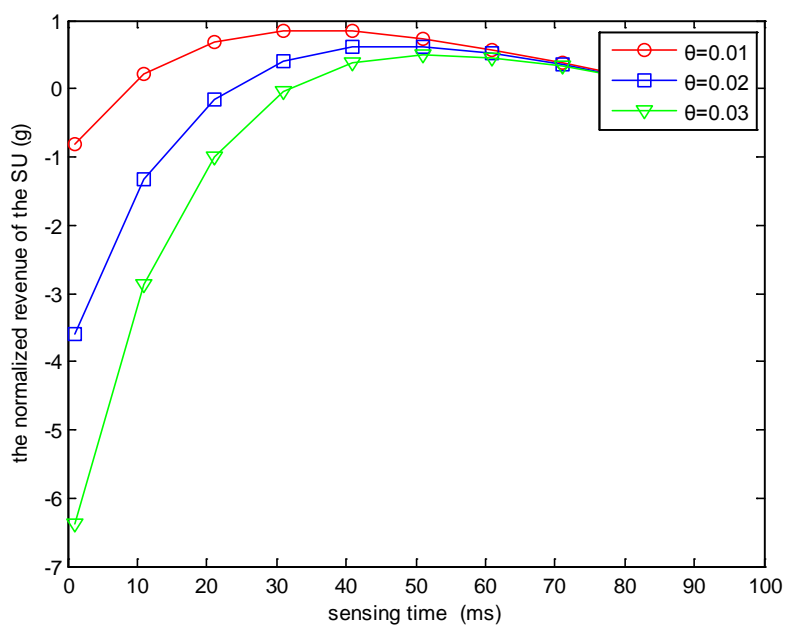

Fig.4. Different $\theta$ 's Influence on the SU's Revenue

The three curves above shows the SU's revenue changes with the variation of sensing time when the price-loss rate is 
set to different values, indicating PU's preference on this kind of collision compensation is relevant to PU's utility rate on the channel. In the situation of $\theta=0.1$, the optimal sensing time is $t 1=32 \mathrm{~ms}$. In the situation of $\theta=0.2$, the optimal sensing time is $t 2=43 \mathrm{~ms}$. In the situation of $\theta=0.3$, the optimal sensing time is $t 3=50 \mathrm{~ms}$. As the price-loss transmission rate increases, shorter sensing time will increase the possibility of wrong sensing result, while the compensation cost increases. The optimal sensing time increases as the transmission rate increases.

At last, we compared PU's revenue variations when collision compensation is introduced into the question.

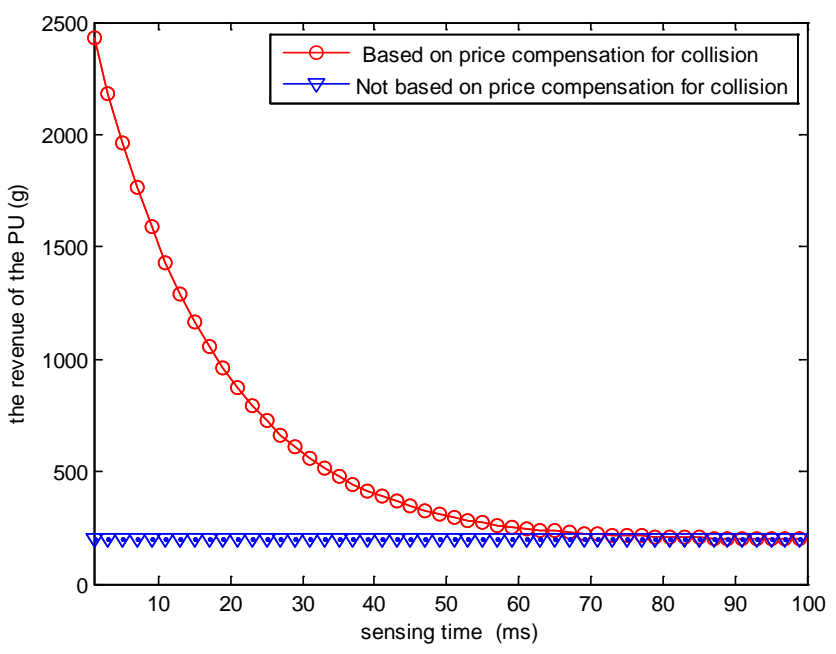

Fig.5.PU's Revenue Variation after Collision Compensation Is Introduced

In Fig. 5, the two curves show the revenue of PU's after collision compensation is introduced. It can be seen that PU's revenue increases on the whole, which is important to the PU's willingness to participate in CR's development. What's more, the longer the sensing time, the smaller the possibility of wrong sensing result, also the smaller the loss it causes PU. Therefore, after the introduction of collision compensation, PU's revenue declines as sensing time increases.

\section{CONCLUSIONS}

In this paper, we discuss the optimization of SU's revenue based on price compensation for collision. In this system, when it senses that the PU does not use its spectrum, the SU can access to the spectrum.

In our scene, the SU is not allowed to transmit data within the sensing time, thus, testing for too long will lead to the SU's transmission time reduced and decline in revenue. If sensing time is too short, the sensing error rate will be increased, resulting in the collision between the PU and the SU. In the end, by the game, we get the optimal result of sensing time allocation which makes the SU's revenue maximum in the collision compensation mechanism. And this algorithm takes into account the compensation for the impact of spectrum collision. What's more, the effects of sensing errors will be introduced into the pricing mechanism, which is helpful to make pricing more in line with reality, and improve the PU's participation enthusiasm to share spectrum. We use the cycle sensing mechanism that in every fixed period the SU conducts a test of the spectrum, which has increased the utility in the field of communications. Moreover, since the SU's final profit is only related to different error rate due to different sensing time, and unrelated to the influence of the time when the PU occupies and exits channel, so that the model has been greatly simplified.

\section{ACKNOWLEDGMENT}

This work is supported in part by Research Innovation Fund for College Students of Beijing University of Posts and Telecommunications , NSFC Project 61531007and 61302083.

\section{References}

[1] Zhao Q and Sadler B, "A survey of dynamic spectrum access: signal processing, networking, and regulatory policy,” IEEE Signal Processing Magazine, vol. 24, no. 3,2007,pp. 79-89.

[2] Haykin S, "Cognitive radio: brain-empowered wireless communications ,” IEEE Journal on Selected Areas in Communications, vol. 23, no. 2, 2005, pp.201-220.

[3] J.Elias, "Joint operator pricing and network selection game in cognitive radio networks: equilibrium, system dynamics and price of anarchy," IEEE Trans. Vehicular Technology, vol. 62, no. 9, Nov. 2013, pp.4576 4589

[4] Jia Y, Zhang Z, Tan X, “Analysis of Spectrum Pricing Framework in Cognitive Networks," Journal of Information and Computational Science, vol.11, no.9, June 10 2014, 3047-3061

[5] Niyato D, Hossain E,"Market-equilibrium, Competitive, and Cooperative Pricing for Spectrum Sharing in Cognitive Radio Networks: Analysis and Comparison," IEEE Transactions on Wireless Communications, vol.11,no.7,2008, pp.4273-4283.

[6] Niyato D, Hossain E, "Competitive Pricing for Spectrum Sharing in Cognitive Radio Networks: Dynamic Game, Inefficiency of Nash Equilibrium, and Collusion,” IEEE Journal on Selected Areas in Communications, vol.26,no.1,2008, pp.192-202.

[7] HJ Wang, “ Cooperative Spectrum Detection Techniques in Cognitive Radio,” ZTE Communications,Feb.2009

[8] Wang P, "Optimization of detection time for channel efficiency in cognitive radio systems,” IEEE WCNC, vol.11, no.15, March 2007, pp.111-115.

[9] Liang Ying-Chang, "Sensing-throughput tradeoff for cognitive radio networks,” IEEE Transactions on Wireless Communications, vol. 7, no. 4, Apr.2008, pp.1326 - 1337

[10] Jia Y, “Asymmetric active cooperation strategy in spectrum sharing game with imperfect information,’'International Journal of Communication Systems, vol.28,no.3, February 2015, pp. 414-425.

[11] Jia Y, "Research on competition and interference avoidance for cognitive radio dynamic spectrum access,” Ph.D. dissertation, Harbin Institute of Technology,China, 2014. 